\title{
On-line monitoring of downstream purification processes for VSV based SARS-CoV-2 vaccine by a novel technique
}

\author{
Arik Makovitzki ${ }^{1}$, Avital Jayson ${ }^{1}$, Ziv Oren ${ }^{1}$, Elad Lerer ${ }^{1}$, Yaron Kafri ${ }^{1}$, Eyal Dor ${ }^{1}$, Lilach \\ Cherry $^{1}$, Hanan Tzadok ${ }^{1}$, Lilach Levin ${ }^{1}$, Ophir Hazan ${ }^{1}$, Irit Simon ${ }^{1}$, Arnon Tal ${ }^{1}$, Meni \\ Girshengorn $^{1}$, and Osnat Rosen ${ }^{1}$ \\ ${ }^{1}$ Israel Institute for Biological Research
}

June 19, 2021

\begin{abstract}
The COVID-19 pandemic caused by Severe Acute Respiratory Syndrome Coronavirus 2 (SARS-CoV-2) increases the need for rapid development of efficient vaccines. Among other vaccines in clinical trials, a recombinant VSV-[?]G-spike vaccine was developed by the Israel Institute for Biological, Chemical and Environmental sciences (IIBR) and is being evaluated. Development of an efficient downstream purification process (DSP) enables advancing the vaccine to clinical trials. The DSP must eliminate impurities, either process- or product -related, to yield sufficient product with high purity, potency, and quality. To acquire critical information on process restrictions and qualities, incorporation of on-line monitoring is vital. Application of on-line monitoring should significantly impact process yield, product quality and economy of the entire process. Here, we describe an on-line monitoring technique that was applied in the DSP of the VSV-[?]G-spike vaccine. The technique is based on determining concentrations of metabolites, nutrients and a host cell protein (HCP) by the automatic chemistry analyzer Cobas Integra 400 Plus. The analysis revealed critical information on process parameters and significantly impacted purification processes. The technique is rapid, easy and efficient. Adaptation of this technique during the purification process improves process yield, product quality and enhances the economy of the entire downstream process of biotechnology and bio pharmaceutical products.
\end{abstract}

\section{Introduction}

Coronavirus disease (COVID-19) is a disease caused by a novel coronavirus closely related to viruses causing Severe Acute Respiratory Syndrome (SARS) and Middle East Respiratory Syndrome (MERS). Similar to the disease caused by the other two viruses, COVID-19 disease mainly comprises symptoms in the lungs, causing cough and fever (Guan et al. 2020). One strategy to limit viral transmission while simultaneously permitting normal conduct of business and social life is to induce a rapid onset of immune protection by administering vaccines against COVID-19.

Different vaccine candidates have already been approved around the world for general or emergency use. As of May 2021, almost 300 vaccines are in clinical trials, all targeting the spike protein of severe acute respiratory syndrome coronavirus type 2 (SARS-CoV-2). Most vaccines are based on either viral vectors or mRNA all of which encode the spike protein (or fragment of it). Other vaccines are based on whole virus (live-attenuated or inactivated), DNA or recombinant protein subunits https://www.who.int/publications/m/item/draftlandscape-of-covid-19-candidate-vaccines. One of the vaccines being tested in clinical trials is being developed by the IIBR. This vaccine is based on the vesicular stomatitis virus (VSV), in which the VSV-G protein has been replaced with the SARS-CoV-2 spike protein (rVSV-[?]G-spike), creating a recombinant replicating 
virus (Yahalom-Ronen et al. 2020) (ClinicalTrials.gov Identifier: NCT04608305). The rVSV-[?]G-spike is produced in Vero cells, grown on Fibra-Cel bioreactors (Cino et al. 2011). The virus is released into the culture medium, and, at the end of the production process, the medium is collected.

To bring rVSV-[?]G-spike to clinical trials, a downstream process (DSP) that will rescue the virus from the medium and is suitable for scale-up is required (Wolf and Reichl 2011; Nestola et al. 2015). The ultimate goal of DSP is to obtain sufficient product with high purity, potency, safety and quality to meet the stringent guidelines of the regulatory authorities. The DSP must eliminate impurities, either process -related (e.g. , DNase, nutrients, extractables, and leachables) or product -related (e.g. , host cell proteins (HCP), metabolites and host cell DNA (hc-DNA)). The DSP that was developed by IIBR for the rVSV[?]G-spike vaccine is a multistep process that comprised endonuclease digestion, depth and membrane filter clarification, chromatographic purification and ultrafiltration (Makovitzki et al. 2021).

One of the main challenges in DSP development is monitoring of impurities removal in each step of the process. Monitoring could have a very significant impact and may extensively improve the total process yield, product quality and enhance the economy of the entire process (Mandenius and Titchener-Hooker 2013). This challenge can be enhanced by incorporating on-line monitoring for acquiring critical information on process parameters and attributes (Flickinger 2013). The ideal on-line monitoring technique should fulfil certain requirements, such as high sensitivity, rapid response time, high accuracy and robustness. Moreover, it should cover a wide dynamic range, a low limit of detection, have minimal recalibration needs and comply with GMP. However, so far, on-line monitoring opportunities are sparsely discussed in downstream processing. We proposed herein a DSP on-line monitoring approach by following the concentration of metabolites, nutrients and an HCP representative by the automatic chemistry analyzer Cobas Integra 400 Plus.

During the virus production process, metabolites, nutrients and HCP, are either consumed or released into the medium. To follow the production process, these substances are monitored. At the end of the virus production process, the medium collected at harvest contains, in addition to the vaccine virus, all of these substances. A DSP process that eliminates process- and product-related impurities, is expected to remove those substances. To follow the clearance of a substance, a sample was taken from each step of DSP, and its concentration was analyzed by the chemistry analyzer. This analyzer has many advantages that make it a suitable candidate as an on-line monitoring technique: it can run up to 400 tests per hour, and has comprehensive testing capabilities; The format of its reagents ("Cobas c pack") standardizes results across integrated laboratory networks; Each Cobas c pack holds all the necessary reagents for up to 800 determinations, maximizing on-board capacity and reducing the need for operator intervention and frequent reloading; The reagents are ready-to-use and have long stability requiring minimal calibration efforts. Each assay uses 2 to $10 \mu \mathrm{L}$ sample and single-use cuvettes to ensure carry over free analytics; Each test has wide dynamic range. Samples and reagents are refrigerated, preventing reagent evaporation and degradation and ensuring long-term on-board stability and long inter-calibration intervals; The barcoded Cobas c packs are automatically handled by the system, reducing the possibility of errors and saving staff time.

In the study reported here, we analyzed the concentration of several metabolites and nutrients in all steps of DSP. The analysis included GlutaMAX (Ala-Gln di-peptide, AQB), lactate (LAC2B), glucose (GLC2B), Ammonia (NH3B) and glutamate (GLU2B). The normalized results taken from three different DSP's are shown in Figure 1. Two of the DSP were done to a harvest obtained after growing cells in multi-trays and the third was from bioreactors. Since the initial concentration of each metabolite/nutrient differed between harvests, all initial/harvest concentrations were set to $100 \%$. The reduction from $100 \%$ harvest concentration is shown for the following DSP steps: Chromatography, Concentrate, and Diafiltration (DF 1-5).

The chromatography process was performed with captocore 700 resin. This resin contains $700 \mathrm{kDa}$ diameter pores which are positively charged (strong anionic exchanger) only inside the pores. Therefore, only anionic molecules which are $<700 \mathrm{kDa}$ (and $>2 \mathrm{kDa}$ ) can be bound to the resin while all others will move with the flow-through. As indicated in Figure 1, after chromatography, most of the metabolites/nutrients remained at the same concentration and were not removed in the chromatography step. As expected, small cationic and uncharged molecules (such as $\mathrm{NH}_{3}$ and glucose) were not bound to the anionic exchange resin. In addition, 
the anionic small molecules (such as glutamate and lactate) did not bind to the resin (despite their charge) because of their small size. On the other hand, a small reduction $\left({ }^{\sim} 15 \%\right)$ in AQB concentration (Ala-Gln di-peptide) was noted, though this molecule is small and uncharged. This small reduction may result from non-specific absorption of this peptide due to hydrophobic interactions.

The next DSP step was Tangential Flow Filtration (TFF), which consists of concentration and buffer exchange (diafiltration) (Negrete et al. 2014). This is an important step in the DSP of rVSV-[?]G-spike to reduce viral harvested volume while increasing viral concentration, achieve solution exchanges and reduce the impurity load for subsequent purification stages. The TFF step uses a $750 \mathrm{kDa}$ hollow fiber and include 5 cycles of diafiltration (DF) against dialysis buffer with similar $\mathrm{pH}$ and conductivity values as the chromatography product. The similar values of the chromatography product and the dialysis buffer make the determination of complete dialysis difficult based on the common parameters such as $\mathrm{pH}$ and conductivity. Therefore, for this process, the chemistry analyzer was used to determine dialysis progress, discover the point of complete dialysis and evaluate vaccine substance purification progress during the TFF process. Based on Figure 1, two points should be noted and elaborated: 1. The similarities between metabolites/nutrients purification efficiencies profiles, and 2. The resemblance between different DSPs.

The TFF process began with a 6 -fold concentration step (from 12 to 2 liters). For all metabolites tested, a large drop in concentration $(80 \%)$ appeared in this step (despite the concentration). In addition, low SD values were observed, despite all the differences between biological processes (cell density, cell metabolism, proliferation, virus production), and the different source of the DSP. In the first diafiltration cycle (DF1), 9095\% of the small molecules were removed. In the second cycle (DF2), 95-97\%, and in the third cycle (DF3) 98$99 \%$ of the small molecules were eliminated. In the fourth and fifth cycles (DF4 and DF5) the concentrations of small molecules were below the detection threshold (Figure 2). The TFF process using $750 \mathrm{kDa}$ hollow fiber filter efficiently removed the small molecules. Therefore, it was concluded that three diafiltration cycles are sufficient to remove the majority of small contaminating molecules from the vaccine substance. In light of these results, it was decided to stop the diafiltration after three cycles. These results clearly demonstrate the utility of the chemistry analyzer for monitoring removal efficiencies of small contaminating molecules, such as metabolites and nutrients, from the pharmaceutical substance. This method enables determination of a diafiltration cycle number which is needed for efficient purification of the vaccine substance at the TFF stage.

Another substance that was analyzed is Lactate dehydrogenase (LDH), a soluble cytosolic enzyme presents in most eukaryotic cells that is released into culture medium upon cell death due to damage to plasma membrane. $\mathrm{LDH}$ is an enzyme from the dehydrogenase family that functions as a catalyst for the redox reaction of lactate and pyruvate. $\mathrm{LDH}$ is defined as the contaminant protein in viruses and recombinant protein drug substances that are produced from cell cultures. In practice, LDH is one of the main HCP's which are produced in the cell culture fermentations (Yuk et al. 2015). Tracking LDH removal in different steps of DSP can help in selecting desired DSP steps and their order of performance. The HCP proteins are defined as contaminating proteins that should be removed from the vaccine substrate during the purification process; hence LDH removal serves as a convenient indicator of HCP removal.

Analysis of LDH concentration was performed with the chemistry analyzer for two different DSPs: one as described above and the other without the chromatography step. As can be seen in Figure 3a, the LDH was efficiently removed in the chromatography stage using the captocore 700 strong anion exchanger (red line). Since $\mathrm{LDH}$ is a $140 \mathrm{kDa}$ anion, its complete removal by the chromatography is expected. In contrast, the TFF removed LDH enzyme only partially (40-60\%), although the LDH molecular weight is much smaller than the TFF cutoff (purple line). This can be explained by the high hydrophobicity of the LDH molecule and its possible binding to other hydrophobic molecules in the vaccine substance, that results in aggregation and, hence, the inability of the TFF to remove LDH from the solution. Since LDH is one of the main HCP's, the purification profiles of LDH and the total HCP in the same DSPs were compared. The analysis of total HCP was done by ELISA. A similar pattern was found for LDH and total HCP removal (Figure $3 \mathrm{~b}$ ). Chromatography by captocore 700 decreased HCP concentration to almost zero (from 100 to $0.6 \%$ ) 
whereas TFF resulted in $\sim 40-50 \%$ clearance. These results validate the application of concentration analysis by chemistry analyzer for on-line monitoring of DSP efficiency.

In summary, we suggest employing an on-line monitoring technique in downstream purification processes. Application of this technique was demonstrated in DSP for VSV based SARS-CoV-2 vaccine. Sample analysis from all purification steps with chemistry analyzer revealed critical information on process parameters and attributes. All small molecule metabolites were removed in the diafiltration steps performed by only 3 diafiltration cycles. The removal of more than $98 \%$ of the metabolites after three diafiltration cycles is critical in meeting guidelines of the regulatory authorities. Having only 3 cycles (and not 5 as initially thought) lowers the duration of the DSP and the expenses involved. The reduction in DF cycles also improves the process yields since the virus is sensitive to continues shear forces that occur while TFF process. Moreover, the same $\mathrm{pH}$ and conductivity of dialysis buffer and chromatography buffer, made it difficult to follow the diafiltration outcome. Sample analysis with chemistry analyzer overcome these limitations. The analysis also showed that LDH was removed completely by chromatography. Altogether, analysis of samples with the chemistry analyzer is ideal as on-line monitoring technique. It is rapid, efficient, sensitive, accurate and requires only minimal recalibrations. Adaptation of this technique during the purification process can improve process yield, product quality and enhance the economy of the entire downstream process of biotechnology and bio pharmaceutical products.

\section{Material and methods}

\section{Virus Upstream production}

The rVSV-[?]G-spike vaccine was produced in BioBLUß) 5p Eppendorf single-use bioreactors (Cino et al. 2011). Vero cells were absorbed to fibra-cel within the bioreactor, and grown for six days. Cells were then infected with the vaccine virus and the virus was allowed to replicate in the cells for 48 hours. The medium from the bioreactor, containing the released vaccine virus, was harvested into a Flexboy bag (Sartorius, Gottingen, Germany). The bag was transferred to the downstream process. Some purification processes were done to harvest medium from multi-tray flasks (instead of bioreactors).

\section{Virus purification}

DSP of rVSV-[?]G-spike comprises the several steps described in (Makovitzki et al. 2021). Briefly, the first step is endonuclease digestion of host cell DNA, followed by clarification by a train of decreasing cut off filters. The next step is chromatographic purification, with AKTA Pilot 600 equipped with captocore 700 resin packed in Index 100/50 column (Cytiva, USA). The last step is tangential flow filtration (TFF) which concentrates and diafiltration using uniflux 10 TFF system equipped with polysulfone (PS) $750 \mathrm{kDa}$ molecular weight cutoff, $1 \mathrm{~mm}$ internal diameter hollowfiber filter (Cytiva, USA).

\section{Chemistry Analyzer Cobas integra 400 plus}

The Cobas Integra 400(r) Plus (Roche Diagnostics GmbH, Mannheim, German) is an automated wet chemistry analyzer with comprehensive testing capabilities. A few tens of samples, taken from different steps of DSP, from different protocols, were tested with the Cobas chemistry analyzer. One hundred $\mu$ l of each sample were inserted into Eppendorf tubes (with no cap) and tested for GlutaMAX (AQB), lactate (LAC2B), glucose (GLC2B), Ammonia (NH3B), glutamate (GLU2B) and LDH using commercially available kits from Roche. The concentration of each sample after harvest was set to $100 \%$ and the other results were normalized accordingly. Average and SD were calculated from 3 different DSP processes. 


\section{Host cell protein (HCP) analysis}

Residual proteins of Vero cells were measured using a Vero cell host cell protein (HCP) ELISA kit (Cygnus Technologies, Southport, NC, USA), following the manufacturer's instructions. A total amount of $50 \mu \mathrm{L}$ of diluted samples were added to microtiter strips coated with an affinity purified capture polyclonal goat antiVero cell antibody. Horseradish peroxidase (HRP) enzyme labeled anti-Vero cell antibody (goat polyclonal, $100 \mu \mathrm{L}$ ) was added to wells and incubated for $2 \mathrm{~h}$ at $25^{\circ} \mathrm{C}$ with shaking at $500 \mathrm{rpm}$. The wells were washed 4 times with wash buffer to remove unbound reactants. An amount of $100 \mu \mathrm{L}$ of $3,3^{\prime}, 5,5^{\prime}$ tetramethylbenzidine (TMB) solution was immediately added to the plate and incubated at $25^{\circ} \mathrm{C}$ for 30 mins. The reaction was stopped using $100 \mu \mathrm{L}$ of $0.5 \mathrm{M} \mathrm{H}_{2} \mathrm{SO}_{4}$ stop solution and the absorbance was read at $450 \mathrm{~nm}$ using CLARIOstar multi-mode reader (BMG LABTECH, Ortenberg, Germany). All standards, controls, and samples were assayed in duplicate. The residual Vero HCP concentration $(\mathrm{ng} / \mathrm{mL})$ in a tested sample was calculated by interpolation from a 4-parameter non-linear fit regression of the standard curve after subtracting the blank.

\section{Acknowledgments}

We would like to thank Dr. Sandy Livnat for editorial assistance.

\section{Conflicts of interest}

The authors have no conflicts of interest to declare that are relevant to the content of this article.

\section{References}

Cino J, Mirro R, Kedzierski S (2011) An Update on the Advantages of Fibra-Cel@ Disks for Cell Culture Flickinger MC (2013) Downstream industrial biotechnology: recovery and purification. John Wiley \& Sons Guan W-J, Ni Z-Y, Hu Y, et al (2020) Clinical Characteristics of Coronavirus Disease 2019 in China. N Engl J Med 382:1708-1720. https://doi.org/10.1056/NEJMoa2002032

Lerer E, Oren Z, Kafri Y, et al (2021) Highly Efficient Purification of Recombinant VSV-[?]G-spike Vaccine Against SARS-CoV-2 By Flow-through Chromatography. Prep

Makovitzki A, Lerer E, Kafri Y, et al (2021) Evaluation of a Downstream Process for the Recovery and Concentration of a Cell-Culture-Derived rVSV-Spike COVID-19 Vaccine Candidate. Prep

Mandenius C-F, Titchener-Hooker NJ (2013) Measurement, monitoring, modelling and control of bioprocesses. Springer

Negrete A, Pai A, Shiloach J (2014) Use of hollow fiber tangential flow filtration for the recovery and concentration of HIV virus-like particles produced in insect cells. J Virol Methods 195:240-246. https://doi.org/https://doi.org/10.1016/j.jviromet.2013.10.017

Nestola P, Peixoto C, Silva RRJS, et al (2015) Improved virus purification processes for vaccines and gene therapy. Biotechnol Bioeng 112:843-857. https://doi.org/10.1002/bit.25545

Wolf MW, Reichl U (2011) Downstream processing of cell culture-derived virus particles. Expert Rev Vaccines 10:1451-1475

Yahalom-Ronen Y, Tamir H, Melamed S, et al (2020) A single dose of recombinant VSV-[?]G-spike vaccine provides protection against SARS-CoV-2 challenge. Nat Commun 11:6402. https://doi.org/10.1038/s41467020-20228-7

Yuk IH, Nishihara J, Walker DJ, et al (2015) More similar than different: Host cell protein production using three null CHO cell lines. Biotechnol Bioeng 112:2068-2083. https://doi.org/10.1002/bit.25615

FIGURE 1 Metabolite and nutrient purification efficiencies for three DSPs. Each metabolite/nutrient was measured in each step of DSP. The initial concentration was set as $100 \%$ and all other were normalized accordingly. The results from three processes were averaged and the SD was calculated. 
FIGURE 2 Metabolites and nutrients purification efficiencies at diafiltration step (Zoom in from Figure 1). The dashed line represents $2 \%$ of the initial concentration.

FIGURE 3 (a) LDH and (b) HCP purification profiles in regular DSP (with chromatography) and in DSP without chromatography step.

FIGURE 1

\section{Hosted file}

image1.emf available at https://authorea.com/users/420580/articles/526911-on-linemonitoring-of-downstream-purification-processes-for-vsv-based-sars-cov-2-vaccine-bya-novel-technique

\section{FIGURE 2}

\section{Hosted file}

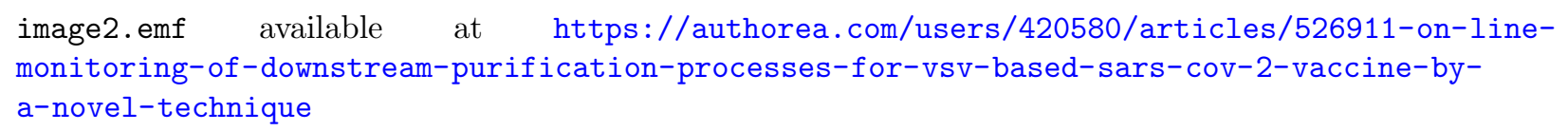

FIGURE 3

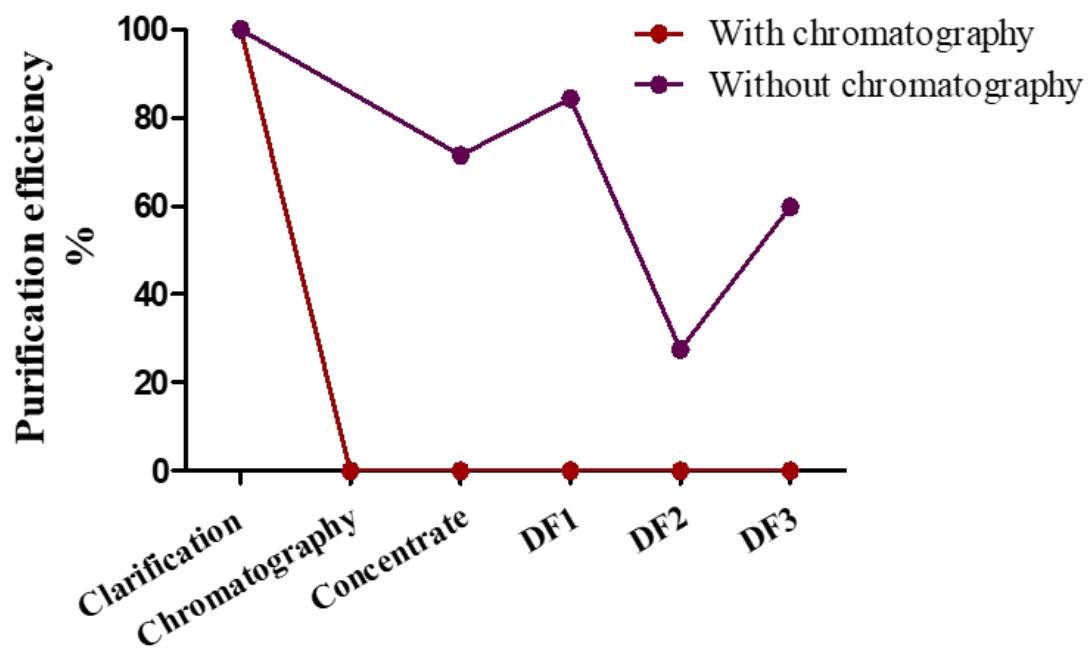

Purification steps 

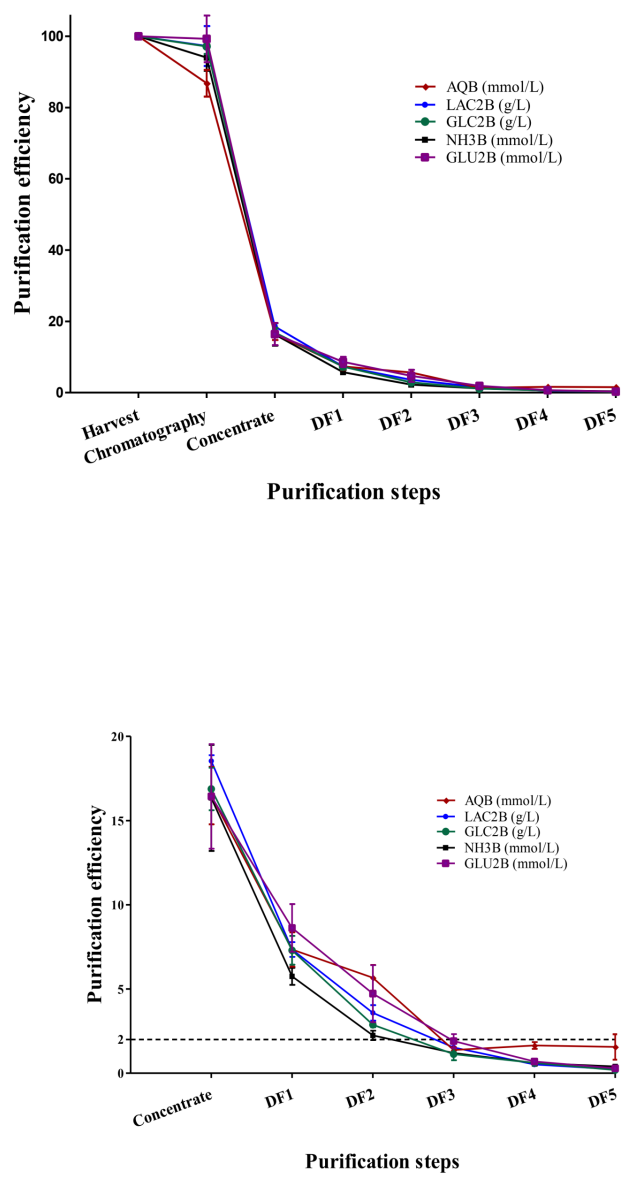

\section{Hosted file}

Fig.3.docx available at https://authorea.com/users/420580/articles/526911-on-linemonitoring-of-downstream-purification-processes-for-vsv-based-sars-cov-2-vaccine-bya-novel-technique 